\title{
Stability of impulsive differential systems with state-dependent impulses via the linear decomposition method
}

\author{
Jingting Hua ${ }^{a}$, Guixia Suib,d, Haydar Akca ${ }^{c}$, Xiaodi Li ${ }^{\mathrm{d}, *}$ \\ ${ }^{a}$ School of Mathematical Sciences, University of Jinan, Ji'nan 250022, P. R. China. \\ ${ }^{b}$ Primary Education Department, Jinan Preschool Education College, Ji'nan 250307, P. R. China. \\ ${ }^{c}$ Department of Mathematics, College of Arts and Science Faculty, Abu Dhabi University, P.O. Box 59911, Abu Dhabi, United Arab \\ Emirates. \\ ${ }^{d}$ School of Mathematics and Statistics, Shandong Normal University, Ji'nan 250014, P. R. China.
}

Communicated by M. Bohner

\begin{abstract}
In this paper, we discuss the stability problem of the impulsive differential systems with state-dependent impulses. By using the linear decomposition methods, some sufficient conditions ensuring stability of the impulsive differential systems with state-dependent impulses are obtained and the estimate of the solution of such nonlinear systems is also acquired. Our results improve and generalize some of the known results given in earlier references. An example is given to demonstrate our results. (C)2017 All rights reserved.
\end{abstract}

Keywords: Stability, impulsive differential systems, state-dependent impulses, linear decomposition methods. 2010 MSC: 34K20, 34K45.

\section{Introduction}

Impulsive differential systems provide basic mathematical models to describe the phenomenon of abrupt changes at certain instants in their states, and the corresponding theory of impulsive differential systems has been rapidly developed in the past decades, see [1, 4, 14, 15, 21]. In general, we can see an impulsive system as a hybrid one which is composed of discrete dynamics, continuous dynamics, criteria for deciding when the states of the system are to be reset. Therefore, an impulsive system has continuous and discrete dynamic behaviors. The complicated dynamic behaviors can be caused because of the interaction of continuous-time dynamics and discrete-time dynamics. Hence, the impulsive system is more efficient than a continuous system or discrete systems in some cases [21]. For example, the savings rates of central bank can not be changed everyday. A deep-space spacecraft can not keep its engine running continuously if it has only a limited supply of fuel. In recent years, the theory of impulsive differential systems has been applied to many aspects including of stability analysis, control design and

\footnotetext{
*Corresponding author

Email address: 1xd@sdnu.edu.cn (Xiaodi Li)
} 
synchronization, etc. A large amount of results on impulsive systems have appeared in the literature, see $[2,5,8,18,22]$.

As we all know, many results for stability of impulsive differential systems with fixed moments of impulse effect have been derived and numerical interesting conclusions have been obtained, see $[3,9-$ $11,13,17,19]$ and references therein. By the method of Lyapunov functionals, the authors in [6, 17] considered the exponential stability of impulsive systems with time delays and an impulsive delay inequality, respectively. Reference [7] discussed further the global stability of periodic solution problem for a class of impulsive neural networks with time delays by using contraction mapping theorem. Some necessary and sufficient conditions were given in [3] for stability of linear impulsive systems with periodic impulses by using continuous-time time-varying discontinuous Lyapunov functions. Based on impulsive control theory and Lyapunov functional techniques, the authors in [9-11] studied the stability properties of nonlinear differential systems with state-dependent delayed impulses, nonlinear systems with delayed impulses and impulsive stochastic functional differential equations, respectively. Furthermore, the problem of stability for impulsive differential systems with state-dependent impulses has gained much research attention. For example, the stability of delay differential equations with state-dependent impulses was studied in [16]. However, there are very few published literatures in related to the stability of impulsive differential systems with state-dependent impulses. Therefore, it requires further studies.

The purpose of this paper is to improve the results in $[16,20]$ and establish some sufficient conditions guaranteeing the stability of impulsive differential systems with state-dependent impulses. Based on the linear decomposition methods, the stability problem of nonlinear systems with state-dependent impulses is converted to that of the linear systems with fixed impulses. The remainder of the paper is organized as follows. In Section 2, we present some basic notations and definitions. In Section 3, we provide the main results. An example is given in Section 4 to demonstrate the feasibility and advantage of the obtained results. In Section 5, conclusions are eventually given.

\section{Preliminaries}

Notations. Let $\mathbb{R}$ be the set of real numbers, $\mathbb{R}_{+}$the set of nonnegative real numbers, $\mathbb{R}^{n}$ the $n$ dimensional real space equipped with the-Euclidean norm $|\cdot|, \mathbb{R}^{n \times m}$ the $n \times m$-dimensional matrix, $\mathbb{Z}_{+}$the set of positive integer numbers, I the $n$-dimensional identity matrix, $\lambda_{\max }(A)$ the maximum eigenvalue of symmetric matrix $A$. For any sets $\mathbb{A}, \mathbb{B} \subseteq \mathbb{R}^{k}(1 \leqslant k \leqslant n), C[\mathbb{A}, \mathbb{B}]=\{\psi: \mathbb{A} \rightarrow \mathbb{B}$ is continuous $\}$. $N\left(t_{0}, t\right)$ denotes the number of impulse times in the semi-open interval $\left[t_{0}, t\right)$.

Consider the following nonlinear impulsive differential system

$$
\begin{cases}\dot{x}(t)=P(t) x+h(t, x), & t \neq \tau_{k}(x), t \geqslant t_{0}, \\ \Delta x=Q_{k} x+u_{k}(x), & t=\tau_{k}(x), \\ x\left(t_{0}\right)=x_{0}, & t_{0} \geqslant 0,\end{cases}
$$

where $x(t) \in \mathbb{R}^{n}$ is the state variable, and $x(t)$ is right continuous, i.e., $x\left(t^{+}\right)=x(t) . P(t) \in \mathbb{R}^{n \times n}$ is bounded and continuous with $t \geqslant t_{0} . Q_{k} \in \mathbb{R}^{n \times n}$ is a constant matrix. $h(t, x): \mathbb{R}_{+} \times \mathbb{R}^{n} \rightarrow \mathbb{R}^{n}$, is continuous in $x$, which satisfies $|x| \leqslant \eta, \eta>0$, and $h(t, x)$ is continuous or piecewise continuous for $t \geqslant t_{0} . \mathfrak{u}_{k}(x): \mathbb{R}^{n} \rightarrow \mathbb{R}^{n}, k \in \mathbb{Z}_{+}$is continuous with $|x| \leqslant \eta . \tau_{k}(x) \in C\left[\mathbb{R}^{n}, \mathbb{R}_{+}\right), \tau_{k}(x)<\tau_{k+1}(x)$ for any $k \in \mathbb{Z}_{+}$, and $\lim _{k \rightarrow \infty} \tau_{k}(x)=\infty$ is uniform in $x . S_{k}: t=\tau_{k}(x)$ denotes the impulsive surface for every $k \in \mathbb{Z}_{+}$.

The corresponding linear system is given as follows

$$
\begin{cases}\dot{x}(t)=P(t) x, & t \neq t_{k}, t \geqslant t_{0} \\ \Delta x=Q_{k} x, & t=t_{k} \\ x\left(t_{0}\right)=x_{0}, & t_{0} \geqslant 0\end{cases}
$$

where $t_{k}$ are some constants satisfying $\left|t_{k}-\tau_{k}(0)\right| \leqslant \varepsilon$ for $|x| \leqslant \eta$, where $\varepsilon=\varepsilon(\eta)>0$ is a given constant satisfying $\lim _{\eta \rightarrow 0} \varepsilon(\eta)=0$. 
Some definitions and lemma used in this paper are given in the following.

Definition $2.1([12])$. Let $x(t)=x\left(t, t_{0}, x_{0}\right)$ be any solution of (2.1). Then the zero solution of system (2.1) is said to be

(S1) stable, if for any $\epsilon>0$ and $t_{0} \geqslant 0$, there exists a constant $\delta=\delta\left(t_{0}, \epsilon\right)>0$, such that $\left|x_{0}\right|<\delta$ implies $|x(t)|<\epsilon, t \geqslant t_{0} ;$

(S2) attractive, if for any $\epsilon>0$ and $t_{0} \geqslant 0$, there exist constants $\delta=\delta\left(t_{0}\right)>0$ and $T\left(\epsilon, t_{0}, x_{0}\right)>0$ such that $\left|x_{0}\right|<\delta$ implies $|x(t)|<\epsilon, t \geqslant t_{0}+T$;

(S3) asymptotically stable, if it is stable and attractive;

(S4) exponentially stable, if for any $\epsilon>0$ and $t_{0} \geqslant 0$, there exist constants $\delta(\epsilon)>0$ and $\lambda>0$, such that $\left|x_{0}\right|<\delta(\epsilon)$ implies $|x(t)|<\epsilon e^{-\lambda\left(t-t_{0}\right)}, t \geqslant t_{0}$.

Lemma 2.2 ([20]). For $\mathrm{t} \geqslant \mathrm{t}_{0}$ let $\mathrm{u}(\mathrm{t})$ be a nonnegative piecewise continuous function satisfying

$$
\mathrm{u}(\mathrm{t}) \leqslant \mathrm{c}+\int_{\mathrm{t}_{0}}^{\mathrm{t}} v(\mathrm{~s}) \mathrm{u}(\mathrm{s}) \mathrm{d} s+\sum_{\mathrm{t}_{0} \leqslant \tau_{i}<\mathrm{t}} \mathrm{b}_{\mathrm{i}} \mathrm{u}\left(\tau_{\mathrm{i}}\right),
$$

where $c \geqslant 0, b_{i} \geqslant 0, v(s)>0, u(t)$ has discontinuous points of the first kind at $\tau_{i}$. Then we have

$$
u(t) \leqslant c \prod_{t_{0}<\tau_{i}<t}\left(1+b_{i}\right) \exp \left(\int_{t_{0}}^{t} v(s) d s\right) .
$$

\section{Main results}

In this section, we shall establish some sufficient conditions ensuring the stability properties of system (2.1).

Theorem 3.1. Suppose that

(i) for any $|x| \leqslant \eta$, and $k \in \mathbb{Z}_{+}$

$$
\tau_{k}\left(\left(I+Q_{k}\right) x+u_{k}(x)\right) \leqslant \tau_{k}(x) \leqslant \tau_{k+1}\left(\left(I+Q_{k}\right) x+u_{k}(x)\right)
$$

(ii) for any $\mathrm{t} \geqslant \mathrm{t}_{0},|x| \leqslant \eta$, and $\mathrm{k} \in \mathbb{Z}_{+}$, we have

$$
|h(t, x)| \leqslant a(t)|x|, \quad\left|u_{k}(x)\right| \leqslant b(t)|x|,
$$

where $\mathrm{a}(\mathrm{t}) \in\left(\mathbb{R}_{+}, \mathbb{R}_{+}\right), \mathrm{b}(\mathrm{t}) \in\left(\mathbb{R}_{+}, \mathbb{R}_{+}\right)$;

(iii) the state transition matrix $\phi(t, s)$ of the linear system (2.2) satisfies

$$
|\phi(t, s)| \leqslant \frac{K}{1+t-s}, \quad K>0, \quad t \geqslant s \geqslant t_{0} ;
$$

(iv) there exists a constant $M>1$ such that

$$
\prod_{t_{0}<t_{k}<t}\left(1+K b\left(t_{k}\right)\right) \exp \left(\int_{t_{0}}^{t} K a(s) d s\right)<M<\infty, \quad t_{k}=\tau_{k}\left(x\left(t_{k}\right)\right) .
$$

Then the zero solution of system (2.1) is asymptotically stable and each solution satisfies

$$
|x(t)| \leqslant \frac{K\left|x_{0}\right| \prod_{t_{0}<t_{k}<t}\left(1+K b\left(t_{k}\right)\right) \exp \left(\int_{t_{0}}^{t} K a(s) d s\right)}{1+t-t_{0}}, t \geqslant t_{0} .
$$


Proof. Let $x(t)=x\left(t, t_{0}, x_{0}\right)$ be any solution of system (2.1) starting at $\left(t_{0}, x_{0}\right)$, where $x_{0}$ is in a small neighborhood of $x=0$. There exist constants $\bar{\eta}<\eta$ and $T \leqslant \infty$ such that $\left|x_{0}\right| \leqslant \bar{\eta}$ implies $|x(t)|<\eta$ for $t \in\left(t_{0}, t_{0}+T\right]$. Based on the condition (i), $x(t)$ meets each surface $t=\tau_{k}(x)$ only once for $t \in\left(t_{0}, t_{0}+T\right]$, then the equation $t=\tau_{k}(x(t))$ has a unique solution denoted by $t_{k}$. Then $x(t), t \in\left(t_{0}, t_{0}+T\right]$ is also a solution of the system as follows

$$
\begin{cases}\dot{x}(t)=P(t) x+h(t, x), & t \neq t_{k}, t \geqslant t_{0} \\ \Delta x=Q_{k} x+u_{k}(x), & t=t_{k} \\ x\left(t_{0}\right)=x_{0}, & t_{0} \geqslant 0\end{cases}
$$

Hence, one can represent $x(t)$ as

$$
x(t)=\phi\left(t, t_{0}\right) x_{0}+\int_{t_{0}}^{t} \phi(t, s) h(s, x(s)) d s+\sum_{t_{0}<t_{k}<t} \phi\left(t, t_{k}\right) u_{k}\left(x\left(t_{k}\right)\right), t \in\left(t_{0}, t_{0}+T\right] .
$$

We can derive that

$$
|x(t)| \leqslant\left|\phi\left(t, t_{0}\right)\right|\left|x_{0}\right|+\int_{t_{0}}^{t}|\phi(t, s)||h(s, x(s))| d s+\sum_{t_{0}<t_{k}<t}\left|\phi\left(t, t_{k}\right)\right|\left|u_{k}\left(x\left(t_{k}\right)\right)\right| .
$$

It then follows from assumptions (ii) and (iii) that

$$
|x(t)| \leqslant \frac{K}{1+t-t_{0}}\left|x_{0}\right|+\int_{t_{0}}^{t} \frac{K}{1+t-s} a(s)|x(s)| d s+\sum_{t_{0}<t_{k}<t} \frac{K}{1+t-t_{k}} b\left(t_{k}\right)\left|x\left(t_{k}\right)\right|,
$$

i.e.,

$$
\left(1+t-t_{0}\right)|x(t)| \leqslant K\left|x_{0}\right|+\int_{t_{0}}^{t} \frac{K\left(1+t-t_{0}\right)}{1+t-s} a(s)|x(s)| d s+\sum_{t_{0}<t_{k}<t} \frac{K\left(1+t-t_{0}\right)}{1+t-t_{k}} b\left(t_{k}\right)\left|x\left(t_{k}\right)\right|,
$$

which leads to

$$
\left(1+t-t_{0}\right)|x(t)| \leqslant K\left|x_{0}\right|+\int_{t_{0}}^{t} K a(s)\left(1+s-t_{0}\right)|x(s)| d s+\sum_{t_{0}<t_{k}<t} K b\left(t_{k}\right)\left(1+t_{k}-t_{0}\right)\left|x\left(t_{k}\right)\right| .
$$

Based on Lemma 2.2, one can get

$$
\left(1+t-t_{0}\right)|x(t)| \leqslant K\left|x_{0}\right| \prod_{t_{0}<t_{k}<t}\left(1+K b\left(t_{k}\right)\right) \exp \left(\int_{t_{0}}^{t} K a(s) d s\right),
$$

i.e.,

$$
|x(t)| \leqslant \frac{K\left|x_{0}\right| \prod_{t_{0}<t_{k}<t}\left(1+K b\left(t_{k}\right)\right) \exp \left(\int_{t_{0}}^{t} K a(s) d s\right)}{1+t-t_{0}} .
$$

If $x_{0}$ satisfies $\left|x_{0}\right|<\frac{\eta}{K M}$ and assumption (iv) holds, one can prove that the zero solution of system (2.1) is asymptotically stable and each solution satisfies

$$
|x(t)| \leqslant \frac{K\left|x_{0}\right| \prod_{t_{0}<t_{k}<t}\left(1+K b\left(t_{k}\right)\right) \exp \left(\int_{t_{0}}^{t} K a(s) d s\right)}{1+t-t_{0}}, t \geqslant t_{0} .
$$


Theorem 3.2. Suppose that

(i) for any $|x| \leqslant \eta$, and $k \in \mathbb{Z}_{+}$

$$
\tau_{k}\left(\left(I+Q_{k}\right) x+u_{k}(x)\right) \leqslant \tau_{k}(x) \leqslant \tau_{k+1}\left(\left(I+Q_{k}\right) x+u_{k}(x)\right) ;
$$

(ii) for any $\mathrm{t} \geqslant \mathrm{t}_{0},|x| \leqslant \eta$ and $\mathrm{k} \in \mathbb{Z}_{+}$, we have

$$
|h(t, x)| \leqslant a(t)|x|, \quad\left|u_{k}(x)\right| \leqslant b(t)|x|,
$$

where $\mathrm{a}(\mathrm{t}) \in\left(\mathbb{R}_{+}, \mathbb{R}_{+}\right), \mathrm{b}(\mathrm{t}) \in\left(\mathbb{R}_{+}, \mathbb{R}_{+}\right)$;

(iii) for any $\mathrm{t} \geqslant \mathrm{t}_{0}$ and $\mathrm{n} \in \mathbb{Z}_{+}$, there exist constants $\mu$ and $v$ such that

$$
\lambda_{\max }\left(\frac{1}{2}\left(\mathrm{P}(\mathrm{t})+\mathrm{P}^{\mathrm{T}}(\mathrm{t})\right)\right)=\lambda_{\mathrm{n}}(\mathrm{t}) \leqslant \mu,
$$

and

$$
\lambda_{\max }\left(\left(\mathrm{I}+\mathrm{Q}_{\mathrm{n}}^{\top}\right)\left(\mathrm{I}+\mathrm{Q}_{\mathrm{n}}\right)\right)=\varpi_{\mathrm{n}}^{2} \leqslant v^{2}
$$

(iv) for any $|x| \leqslant \eta$ the following limit

$$
\lim _{T \rightarrow \infty} \frac{N(t, t+T)}{T}=p
$$

exists and is uniform for $\mathrm{t} \geqslant \mathrm{t}_{0}$;

(v) there exist constants $M>1$ and $\delta>0$ such that

$$
\prod_{t_{0}<t_{k}<t}\left(1+K b\left(t_{k}\right)\right) \exp \left(\int_{t_{0}}^{t} K a(s) d s\right)<M \exp \left(\delta\left(t-t_{0}\right)\right), \quad K \geqslant 1, \quad t_{k}=\tau_{k}\left(x\left(t_{k}\right)\right) ;
$$

(vi) $\mu+p \ln v<0$.

Then the zero solution of system (2.1) is exponentially stable and each solution satisfies

$$
|x(t)| \leqslant K\left|x_{0}\right| \prod_{t_{0}<t_{k}<t}\left(1+K b\left(t_{k}\right)\right) \exp \left(\int_{t_{0}}^{t} K a(s) d s\right) \exp \left(-\alpha\left(t-t_{0}\right)\right), \quad \alpha>0, \quad t \geqslant t_{0} .
$$

Proof. Let $x(t)=x\left(t, t_{0}, x_{0}\right)$ be any solution of system (2.1) starting at $\left(t_{0}, x_{0}\right)$, where $x_{0}$ is in a small neighborhood of $x=0$. There exist constants $\bar{\eta}<\eta$ and $T \leqslant \infty$ such that $\left|x_{0}\right| \leqslant \bar{\eta}$ implies $|x(t)|<\eta$ for $t \in\left(t_{0}, t_{0}+T\right]$. Based on the condition (i), $x(t)$ meets each surface $t=\tau_{k}(x)$ only once for $t \in\left(t_{0}, t_{0}+T\right]$, then the equation $t=\tau_{k}(x(t))$ has a unique solution denoted by $t_{k}$. Thus, $x(t)$ is also a solution of the system (3.1). Then $x(t)$ can be represented as

$$
x(t)=\phi\left(t, t_{0}\right) x_{0}+\int_{t_{0}}^{t} \phi(t, s) h(s, x(s)) d s+\sum_{t_{0}<t_{k}<t} \phi\left(t, t_{k}\right) u_{k}\left(x\left(t_{k}\right)\right), t \in\left(t_{0}, t_{0}+T\right],
$$

where $\phi(t, s)$ is the state transition matrix of linear system (2.2). Let $\widetilde{x}(t)$ be an arbitrary solution of system (2.2) starting at $\left(t_{0}, x_{0}\right)$. Setting $V(t)=\widetilde{x}^{2}(t)=\widetilde{x}^{\top}(t) \widetilde{x}(t), t \in\left[t_{k}, t_{k+1}\right), k \in \mathbb{Z}_{+}$, it follows that

$$
\dot{V}(t)=2 \widetilde{x}^{\top}(t) P(t) \widetilde{x}(t)=\widetilde{x}^{\top}(t)\left(P(t)+P^{\top}(t)\right) \widetilde{x}(t) \leqslant 2 \lambda_{n}(t)\left(\widetilde{x}^{\top}(t) \widetilde{x}(t)\right)=2 \lambda_{n}(t) V(t), t \in\left[t_{k}, t_{k+1}\right), k \in \mathbb{Z}_{+} .
$$


Then we have

$$
V(t) \leqslant V\left(t_{0}\right) \exp \left(2 \int_{t_{0}}^{t} \lambda_{n}(s) d s\right) \leqslant V\left(t_{0}\right) \exp \left(2 \mu\left(t-t_{0}\right)\right), \quad t \in\left[t_{0}, t_{1}\right)
$$

i.e.,

$$
|\widetilde{x}(t)| \leqslant\left|x_{0}\right| \exp \left(\mu\left(t-t_{0}\right)\right), \quad t \in\left[t_{0}, t_{1}\right) .
$$

Note that

$$
V\left(t_{1}\right)=\widetilde{x}^{\top}\left(t_{1}\right) \widetilde{x}\left(t_{1}\right)=\widetilde{x}^{\top}\left(t_{1}^{-}\right)\left(I+Q_{1}\right)^{\top}\left(I+Q_{1}\right) \widetilde{x}\left(t_{1}^{-}\right) \leqslant v^{2} V\left(t_{1}^{-}\right) \leqslant v^{2} V\left(t_{0}\right) \exp \left(2 \mu\left(t_{1}-t_{0}\right)\right),
$$

we can deduce that

$$
\begin{aligned}
V(t) & \leqslant V\left(t_{1}\right) \exp \left(2 \mu\left(t-t_{1}\right)\right) \\
& \leqslant v^{2} V\left(t_{0}\right) \exp \left(2 \mu\left(t_{1}-t_{0}\right)\right) \exp \left(2 \mu\left(t-t_{1}\right)\right) \\
& =v^{2} V\left(t_{0}\right) \exp \left(2 \mu\left(t-t_{0}\right)\right), \quad t \in\left[t_{1}, t_{2}\right),
\end{aligned}
$$

i.e.,

$$
|\widetilde{x}(t)| \leqslant v\left|x_{0}\right| \exp \left(\mu\left(t-t_{0}\right)\right), \quad t \in\left[t_{1}, t_{2}\right) .
$$

Proceeding as before, we obtain

$$
|\widetilde{x}(t)| \leqslant\left|x_{0}\right| v^{N\left(t_{0}, t\right)} \exp \left(\mu\left(t-t_{0}\right)\right), \quad t \in\left[t_{N\left(t_{0}, t\right)}, t_{N\left(t_{0}, t\right)+1}\right) .
$$

In view of assumption (iv), for any $\epsilon>0$ we have

$$
(p-\epsilon)\left(t-t_{0}\right) \leqslant N\left(t_{0}, t\right) \leqslant(p+\epsilon)\left(t-t_{0}\right) .
$$

Thus, we can get

$$
|\widetilde{x}(t)| \leqslant v^{(p \pm \epsilon)\left(t-t_{0}\right)}\left|x_{0}\right| \exp \left(\mu\left(t-t_{0}\right)\right)=\left|x_{0}\right| \exp \left((\mu+(p \pm \epsilon) \ln v)\left(t-t_{0}\right)\right) .
$$

Considering assumption (vi), we can obtain that there exist $K \geqslant 1$ and $\alpha>0$ with $0<\alpha<|\mu+p \ln v|$ such that for any $t_{0} \leqslant s \leqslant t \leqslant t+T$,

$$
|\phi(t, s) Z| \leqslant K \exp (-\alpha(t-s))|Z|,|Z| \leqslant \eta .
$$

Hence, we have

$$
\begin{aligned}
|x(t)| \leqslant & \left|\phi\left(t, t_{0}\right)\right|\left|x_{0}\right|+\int_{t_{0}}^{t}|\phi(t, s)||h(s, x(s))| d s+\sum_{t_{0}<t_{k}<t}\left|\phi\left(t, t_{k}\right)\right|\left|u_{k}\left(x\left(t_{k}\right)\right)\right| \\
\leqslant & K \exp \left(-\alpha\left(t-t_{0}\right)\right)\left|x_{0}\right|+\int_{t_{0}}^{t} K \exp (-\alpha(t-s)) a(s)|x(s)| d s \\
& +\sum_{t_{0}<t_{k}<t} K \exp \left(-\alpha\left(t-t_{k}\right)\right) b\left(t_{k}\right)\left|x\left(t_{k}\right)\right|
\end{aligned}
$$


i.e.,

$$
\begin{aligned}
\exp \left(\alpha\left(t-t_{0}\right)\right)|x(t)| \leqslant & K\left|x_{0}\right|+\int_{t_{0}}^{t} K a(s) \exp \left(\alpha\left(s-t_{0}\right)\right)|x(s)| d s \\
& +\sum_{t_{0}<t_{k}<t} K b\left(t_{k}\right) \exp \left(\alpha\left(t_{k}-t_{0}\right)\right)\left|x\left(t_{k}\right)\right| .
\end{aligned}
$$

Based on Lemma 2.2, it is easy to derive that

$$
|x(t)| \leqslant K\left|x_{0}\right| \prod_{t_{0}<t_{k}<t}\left(1+K b\left(t_{k}\right)\right) \exp \left(\int_{t_{0}}^{t} K a(s) d s\right) \exp \left(-\alpha\left(t-t_{0}\right)\right) .
$$

If $\left|x_{0}\right|<\frac{\eta}{K M}, \delta<\alpha$ and assumption (v) hold, we can derive that the zero solution of system (2.1) is exponentially stable and each solution satisfies

$$
|x(t)| \leqslant K\left|x_{0}\right| \prod_{t_{0}<t_{k}<t}\left(1+K b\left(t_{k}\right)\right) \exp \left(\int_{t_{0}}^{t} K a(s) d s\right) \exp \left(-\alpha\left(t-t_{0}\right)\right), \quad t \geqslant t_{0} .
$$

Theorem 3.3. Suppose that

(i) for any $|x| \leqslant \eta$, and $k \in \mathbb{Z}_{+}$

$$
\tau_{k}\left(\left(I+Q_{k}\right) x+u_{k}(x)\right) \leqslant \tau_{k}(x) \leqslant \tau_{k+1}\left(\left(I+Q_{k}\right) x+u_{k}(x)\right)
$$

(ii) for any $\mathrm{t} \geqslant \mathrm{t}_{0},|x| \leqslant \eta$, and $\mathrm{k} \in \mathbb{Z}_{+}$, we have

$$
|h(t, x)| \leqslant a(t)|x|, \quad\left|u_{k}(x)\right| \leqslant b(t)|x|,
$$

where $\mathrm{a}(\mathrm{t}) \in\left(\mathbb{R}_{+}, \mathbb{R}_{+}\right), \mathrm{b}(\mathrm{t}) \in\left(\mathbb{R}_{+}, \mathbb{R}_{+}\right)$;

(iii) for any $\mathrm{t} \geqslant \mathrm{t}_{0}$ and $\mathrm{n} \in \mathbb{Z}_{+}$, there exist constants $\mu$ and $v$ such that

$$
\lambda_{\max }\left(\frac{1}{2}\left(\mathrm{P}(\mathrm{t})+\mathrm{P}^{\mathrm{T}}(\mathrm{t})\right)\right)=\lambda_{\mathrm{n}}(\mathrm{t}) \leqslant \mu,
$$

and

$$
\lambda_{\max }\left(\left(I+Q_{n}^{\top}\right)\left(I+Q_{n}\right)\right)=\varpi_{n}^{2} \leqslant v^{2}
$$

(iv) for any $k \in \mathbb{Z}_{+}$there exist constants $\xi_{1}$ and $\xi_{2}$ such that

$$
0<\xi_{1} \leqslant \min _{|x| \leqslant \eta} \tau_{k+1}(x)-\max _{|x| \leqslant \eta} \tau_{k}(x) \leqslant \xi_{2},
$$

or else

$$
0<\xi_{1} \leqslant \min _{|x| \leqslant \eta} \tau_{1}(x)-t_{0} \leqslant \xi_{2}
$$

(v) $\mu+\frac{1}{\xi} \ln v<0$, where $\xi=\xi_{1}$ if $v \geqslant 1$, or else $\xi=\xi_{2}$ if $0<v<1$;

(vi) there exist constants $M>1$ and $\delta>0$ such that

$$
\prod_{t_{0}<t_{k}<t}\left(1+K b\left(t_{k}\right)\right) \exp \left(\int_{t_{0}}^{t} K a(s) d s\right)<M \exp \left(\delta\left(t-t_{0}\right)\right), \quad K \geqslant 1, t_{k}=\tau_{k}\left(x\left(t_{k}\right)\right) .
$$


Then the zero solution of system (2.1) is exponentially stable and each solution satisfies

$$
|x(t)| \leqslant K\left|x_{0}\right| \prod_{t_{0}<t_{k}<t}\left(1+K b\left(t_{k}\right)\right) \exp \left(\int_{t_{0}}^{t} K a(s) d s\right) \exp \left(-\alpha\left(t-t_{0}\right)\right), \quad \alpha>0, \quad t \geqslant t_{0} .
$$

Proof. Let $x(t)=x\left(t, t_{0}, x_{0}\right)$ be any solution of system (2.1) starting at $\left(t_{0}, x_{0}\right)$, where $x_{0}$ is in a small neighborhood of $x=0$. There exist constants $\bar{\eta}<\eta$ and $T \leqslant \infty$ such that $\left|x_{0}\right| \leqslant \bar{\eta}$ implies $|x(t)|<\eta$ for $t \in\left(t_{0}, t_{0}+T\right]$. Based on the condition (i), $x(t)$ meets each surface $t=\tau_{k}(x)$ only once for $t \in\left(t_{0}, t_{0}+T\right]$, then the equation $t=\tau_{k}(x(t))$ has a unique solution denoted by $t_{k}$. Thus, $x(t)$ is also a solution of the system (3.1). Then $x(t)$ can be represented as

$$
x(t)=\phi\left(t, t_{0}\right) x_{0}+\int_{t_{0}}^{t} \phi(t, s) h(s, x(s)) d s+\sum_{t_{0}<t_{k}<t} \phi\left(t, t_{k}\right) u_{k}\left(x\left(t_{k}\right)\right), t \in\left(t_{0}, t_{0}+T\right],
$$

where $\phi(t, s)$ is the state transition matrix of linear system (2.2). Let $\widetilde{x}(t)$ be an arbitrary solution of system (2.2) starting at $\left(t_{0}, x_{0}\right)$. Arguing as Theorem 3.2, we obtain

$$
|\widetilde{x}(t)| \leqslant\left|x_{0}\right| v^{N\left(t_{0}, t\right)} \exp \left(\mu\left(t-t_{0}\right)\right), \quad t \in\left[t_{N\left(t_{0}, t\right)}, t_{N\left(t_{0}, t\right)+1}\right) .
$$

In view of assumption (iv), there exists a constant $m \in \mathbb{Z}_{+}$such that

$$
\xi_{1} N\left(t_{0}, t\right) \leqslant t-t_{0} \leqslant \xi_{2}\left[N\left(t_{0}, t\right)+m\right] .
$$

Hence, we have

$$
|\widetilde{x}(t)| \leqslant\left|x_{0}\right| \exp \left(\left(\mu+\frac{1}{\xi_{1}} \ln v\right)\left(t-t_{0}\right)\right), \quad v \geqslant 1
$$

Or else,

$$
|\widetilde{x}(t)| \leqslant \frac{1}{v^{m}}\left|x_{0}\right| \exp \left(\left(\mu+\frac{1}{\xi_{2}} \ln v\right)\left(t-t_{0}\right)\right), \quad 0<v<1 .
$$

Considering assumption (v), we obtain that there exist $K \geqslant 1$ and $\alpha>0$ with $0<\alpha<\left|\mu+\frac{1}{\xi} \ln v\right|$ such that for any $t_{0} \leqslant s \leqslant t \leqslant t+T$,

$$
|\phi(t, s) Z| \leqslant K \exp (-\alpha(t-s))|Z|,|Z| \leqslant \eta .
$$

Then arguing as Theorem 3.2, we have

$$
|x(t)| \leqslant K\left|x_{0}\right| \prod_{t_{0}<t_{k}<t}\left(1+K b\left(t_{k}\right)\right) \exp \left(\int_{t_{0}}^{t} K a(s) d s\right) \exp \left(-\alpha\left(t-t_{0}\right)\right) .
$$

If $\left|x_{0}\right|<\frac{\eta}{K M}, \delta<\alpha$ and assumption (vi) hold, one can derive that the zero solution of system (2.1) is exponentially stable and each solution satisfies

$$
|x(t)| \leqslant K\left|x_{0}\right| \prod_{t_{0}<t_{k}<t}\left(1+K b\left(t_{k}\right)\right) \exp \left(\int_{t_{0}}^{t} K a(s) d s\right) \exp \left(-\alpha\left(t-t_{0}\right)\right), \quad t \geqslant t_{0} .
$$

Next result is concerned with stability of the following system

$$
\begin{cases}\dot{x}(t)=P x+h(t, x), & t \neq \tau_{k}(x), t \geqslant t_{0}, \\ \Delta x=Q x+u_{k}(x), & t=\tau_{k}(x) \\ x\left(t_{0}\right)=x_{0}, & t_{0} \geqslant 0\end{cases}
$$

where $P, Q \in \mathbb{R}^{n \times n}$ are constant matrices. 
Theorem 3.4. Suppose that

(i) for any $|x| \leqslant \eta$, and $k \in \mathbb{Z}_{+}$

$$
\tau_{k}\left((I+Q) x+u_{k}(x)\right) \leqslant \tau_{k}(x) \leqslant \tau_{k+1}\left((I+Q) x+u_{k}(x)\right) ;
$$

(ii) for any $\mathrm{t} \geqslant \mathrm{t}_{0},|\mathrm{x}| \leqslant \eta$ and $\mathrm{k} \in \mathbb{Z}_{+}$, we have

$$
|h(t, x)| \leqslant a(t)|x|, \quad\left|u_{k}(x)\right| \leqslant b(t)|x|,
$$

where $\mathrm{a}(\mathrm{t}) \in\left(\mathbb{R}_{+}, \mathbb{R}_{+}\right), \mathrm{b}(\mathrm{t}) \in\left(\mathbb{R}_{+}, \mathbb{R}_{+}\right)$;

(iii) for any $|x| \leqslant \eta$ the following limit

$$
\lim _{T \rightarrow \infty} \frac{N(t, t+T)}{T}=p
$$

exists and is uniform for $\mathrm{t} \geqslant \mathrm{t}_{0}$;

(iv) $\mu=\max _{i=1}^{n} \operatorname{Re} \lambda_{i}(P), v^{2}=\max _{i=1}^{n} \operatorname{Re} \lambda_{i}\left[\left(I+Q^{\top}\right)(I+Q)\right]$;

(v) there exist constants $M>1$ and $\delta>0$ such that

$$
\prod_{t_{0}<t_{k}<t}\left(1+K b\left(t_{k}\right)\right) \exp \left(\int_{t_{0}}^{t} K a(s) d s\right)<M \exp \left(\delta\left(t-t_{0}\right)\right), \quad K \geqslant 1, \quad t_{k}=\tau_{k}\left(x\left(t_{k}\right)\right) ;
$$

(vi) $\mu+p \ln v<0$.

Then the zero solution of system (3.2) is exponentially stable and each solution satisfies

$$
|x(t)| \leqslant K\left|x_{0}\right| \prod_{t_{0}<t_{k}<t}\left(1+K b\left(t_{k}\right)\right) \exp \left(\int_{t_{0}}^{t} K a(s) d s\right) \exp \left(-\alpha\left(t-t_{0}\right)\right), \quad \alpha>0, \quad t \geqslant t_{0} .
$$

Proof. Let $x(t)=x\left(t, t_{0}, x_{0}\right)$ be any solution of system (2.1) starting at $\left(t_{0}, x_{0}\right)$, where $x_{0}$ is in a small neighborhood of $x=0$. There exist constants $\bar{\eta}<\eta$ and $T \leqslant \infty$ such that $\left|x_{0}\right| \leqslant \bar{\eta}$ implies $|x(t)|<\eta$ for $t \in\left(t_{0}, t_{0}+T\right]$. Based on the condition (i), $x(t)$ meets each surface $t=\tau_{k}(x)$ only once for $t \in\left(t_{0}, t_{0}+T\right]$, then the equation $t=\tau_{k}(x(t))$ has a unique solution denoted by $t_{k}$. Then $x(t)$ is also a solution of system as follows

$$
\begin{cases}\dot{x}(t)=P x+h(t, x), & t \neq t_{k}, t \geqslant t_{0} \\ \Delta x=Q x+u_{k}(x), & t=t_{k} \\ x\left(t_{0}\right)=x_{0}, & t_{0} \geqslant 0\end{cases}
$$

Thus, $x(t)$ can be represented as

$$
x(t)=\phi\left(t, t_{0}\right) x_{0}+\int_{t_{0}}^{t} \phi(t, s) h(s, x(s)) d s+\sum_{t_{0}<t_{k}<t} \phi\left(t, t_{k}\right) u_{k}\left(x\left(t_{k}\right)\right), t \in\left(t_{0}, t_{0}+T\right],
$$

where $\phi(t, s)$ is the state transition matrix of the following linear system

$$
\left\{\begin{array}{l}
\dot{x}(t)=P x, \quad t \neq t_{k}, t \geqslant t_{0} \\
\Delta x=Q x, \quad t=t_{k} \\
x\left(t_{0}\right)=x_{0}, \quad t_{0} \geqslant 0
\end{array}\right.
$$


Let $\widetilde{x}(t)$ be an arbitrary solution of system (3.3) starting at $\left(t_{0}, x_{0}\right)$. Setting $V(t)=\widetilde{x}^{2}(t)=\widetilde{x}^{\top}(t) \widetilde{x}(t)$, $\mathrm{t} \in\left[\mathrm{t}_{\mathrm{k}}, \mathrm{t}_{\mathrm{k}+1}\right), \mathrm{k} \in \mathbb{Z}_{+}$, it follows that

$$
\dot{V}(t)=2 \widetilde{x}^{\top}(t) P \widetilde{x}(t)=\widetilde{x}^{\top}(t)\left(P+P^{\top}\right) \widetilde{x}(t) \leqslant 2 \mu\left(\widetilde{x}^{\top}(t) \widetilde{x}(t)\right)=2 \mu V(t), \quad t \in\left[t_{k}, t_{k+1}\right), \quad k \in \mathbb{Z}_{+} .
$$

Then arguing as Theorem 3.2, we have

$$
|\widetilde{x}(t)| \leqslant\left|x_{0}\right| v^{N\left(t_{0}, t\right)} \exp \left(\mu\left(t-t_{0}\right)\right), \quad t \in\left[t_{N\left(t_{0}, t\right)}, t_{N\left(t_{0}, t\right)+1}\right) .
$$

Proceeding as Theorem 3.2, the proof is complete.

\section{Example}

Example 4.1. Consider the following impulsive system

$$
\begin{cases}\dot{x}(t)=-\frac{1}{1+t} x+\frac{1}{1+t^{2}} x, & t \neq \tau_{i}(x), t \geqslant t_{0}, \\ \Delta x=-\frac{1}{2} x+\left(\frac{1}{3}-\frac{1}{3+t^{2}}\right) x, & t=\tau_{i}(x), \\ x\left(t_{0}\right)=x_{0}, & t_{0} \geqslant 0,\end{cases}
$$

where $\tau_{i}(x)=|x|+i, i \in \mathbb{Z}_{+}$. Note that $x\left(t_{i}^{+}\right)=x\left(t_{i}\right)=\left(I+Q_{i}\right) x\left(t_{i}^{-}\right)+u_{i}\left(x\left(t_{i}^{-}\right)\right)$, one can choose $\mathrm{Q}_{i}=-\frac{1}{2}, \mathrm{u}_{\mathrm{i}}(\mathrm{x})=\mathrm{b}(\mathrm{t}) \mathrm{x}, \mathrm{b}(\mathrm{t})=\frac{1}{3}-\frac{1}{3+\mathrm{t}^{2}}$ and $\eta=2$.

It then follows that

$$
\begin{aligned}
\tau_{i}\left(x\left(t_{i}^{+}\right)\right) & =\tau_{i}\left(\left(I+Q_{i}\right) x\left(t_{i}^{-}\right)+u_{i}\left(x\left(t_{i}^{-}\right)\right)\right) \\
& =\left|\left(1-\frac{1}{2}\right) x\left(t_{i}^{-}\right)+\left(\frac{1}{3}-\frac{1}{3+t^{2}}\right) x\left(t_{i}^{-}\right)\right|+i \\
& =\left|\left(\frac{5}{6}-\frac{1}{3+t^{2}}\right)\right|\left|x\left(t_{i}^{-}\right)\right|+i \\
& \leqslant \frac{5}{6}\left|x\left(t_{i}^{-}\right)\right|+i \\
& \leqslant\left|x\left(t_{i}^{-}\right)\right|+i \\
& =\tau_{i}\left(x\left(t_{i}^{-}\right)\right)
\end{aligned}
$$

and

$$
\begin{aligned}
& \tau_{i+1}\left(\left(I+Q_{i}\right) x\left(t_{i}^{-}\right)+u_{i}\left(x\left(t_{i}^{-}\right)\right)\right) \\
&=\left|\left(1-\frac{1}{2}\right) x\left(t_{i}^{-}\right)+\left(\frac{1}{3}-\frac{1}{3+t^{2}}\right) x\left(t_{i}^{-}\right)\right|+i+1 \\
&=\left|\left(\frac{5}{6}-\frac{1}{3+t^{2}}\right)\right|\left|x\left(t_{i}^{-}\right)\right|+i+1 \\
& \geqslant \frac{1}{2}\left|x\left(t_{i}^{-}\right)\right|+i+1 \\
& \geqslant\left|x\left(t_{i}^{-}\right)\right|+i \\
&=\tau_{i}\left(x\left(t_{i}^{-}\right)\right) .
\end{aligned}
$$

Choose $h(t, x)=a(t) x, a(t)=\frac{1}{1+t^{2}}$, then it is easy to get that

$$
|h(t, x)| \leqslant \frac{1}{1+t^{2}}|x| .
$$


Note that $u_{i}(x)=\left(\frac{1}{3}-\frac{1}{3+t^{2}}\right) x$, then one can derive that

$$
\left|u_{i}(x)\right| \leqslant\left(\frac{1}{3}-\frac{1}{3+t^{2}}\right)|x| .
$$

The corresponding linear system of (4.1) is given as follows

$$
\begin{cases}\dot{x}(t)=-\frac{1}{1+t} x, & t \neq t_{i}, t \geqslant t_{0}, \\ \Delta x=-\frac{1}{2} x, & t=t_{i}, \\ x\left(t_{0}\right)=x_{0}, & t_{0} \geqslant 0 .\end{cases}
$$

Let $\widetilde{x}(t)$ be the solution of linear system (4.2) satisfying

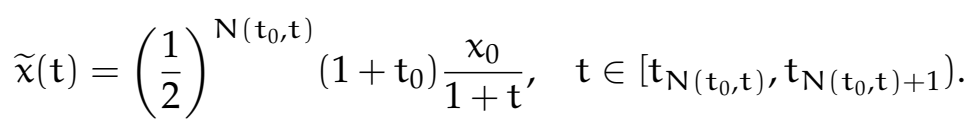

We have

$$
|\widetilde{x}(t)| \leqslant \frac{K}{1+t-s}, \quad t \geqslant s \geqslant t_{0},
$$

where $0<\frac{1}{2}\left(1+t_{0}\right)\left|x_{0}\right|=K<\infty$.

Furthermore, notice that

$$
\prod_{\mathrm{t}_{0}<\mathrm{t}_{\mathrm{i}}<\mathrm{t}}\left(1+\mathrm{Kb}\left(\mathrm{t}_{\mathrm{i}}\right)\right)=\prod_{\mathrm{t}_{0}<\mathrm{t}_{\mathrm{i}}<\mathrm{t}}\left(1+\mathrm{K}\left(\frac{1}{3}-\frac{1}{3+\mathrm{t}_{\mathrm{i}}^{2}}\right)\right),
$$

and

$$
\exp \left(\int_{t_{0}}^{t} K a(s) d s\right)=\exp \left(\int_{t_{0}}^{t} K \frac{1}{1+s^{2}} d s\right),
$$

are convergent, which implies

$$
\prod_{\mathrm{t}_{0}<\mathrm{t}_{i}<\mathrm{t}}\left(1+\mathrm{Kb}\left(\mathrm{t}_{\mathrm{i}}\right)\right) \exp \left(\int_{\mathrm{t}_{0}}^{\mathrm{t}} \mathrm{Ka}(\mathrm{s}) \mathrm{d} s\right)<M<\infty, \quad \mathrm{t}_{\mathrm{i}}=\tau_{\mathrm{i}}\left(x\left(\mathrm{t}_{\mathrm{i}}\right)\right) .
$$

Then it is not difficult to check that conditions (i)-(iv) are satisfied. Thus, we can get from Theorem 3.1 that the zero solution of system (4.1) is asymptotically stable and each solution satisfies

$$
|x(t)| \leqslant \frac{K\left|x_{0}\right| \prod_{t_{0}<t_{i}<t}\left(1+K b\left(t_{i}\right)\right) \exp \left(\int_{t_{0}}^{t} K a(s) d s\right)}{1+t-t_{0}}, t \geqslant t_{0} .
$$

\section{Conclusion}

We have studied the stability properties of impulsive differential systems with state-dependent impulses. Based on the linear decomposition methods, some sufficient conditions guaranteeing asymptotical and exponential stabilities of the impulsive differential systems with state-dependent impulses have been presented. Our results are more general than those mentioned in the literature. An example was given to show the effectiveness of the obtained results. It would be interesting to further extend the approach in this paper to address some nonlinear systems. 


\section{Acknowledgment}

This work was supported by National Natural Science Foundation of China $(61673247,11301308)$ and the outstanding Youth Foundation of Shandong Province (ZR2016JL024, JQ201719).

\section{References}

[1] D. D. Baĭnov, P. S. Simeonov, Systems with Impulse Effect: Stability Theory and Applications, John Wiley \& Sons, New York, (1989). 1

[2] W.-H. Chen, W. X. Zheng, Exponential stability of nonlinear time-delay systems with delayed impulse effects, Automatica J. IFAC, 47 (2011), 1075-1083. 1

[3] B. Corentin, Convex conditions for robust stability analysis and stabilization of linear aperiodic impulsive and sampled-data systems under dwell-time constraints, Automatica J. IFAC, 49 (2013), 3449-3457. 1

[4] V. Lakshmikantham, D. D. Bănov, P. S. Simeonov, Theory of impulsive differential equations, World Scientific, Singapore, (1989). 1

[5] X. Li, M. Bohner, C.-K. Wang, Impulsive differential equations: Periodic solutions and applications, Automatica J. IFAC, 52 (2015), 173-178. 1

[6] X. Li, J. Cao, An impulsive delay inequality involving unbounded time-varying delay and applications, IEEE Trans. Automat. Control, 62 (2017), 3618-3625. 1

[7] X. Li, S. Song, Impulsive control for existence, uniqueness andglobal stability of periodic solutions of recurrentneural networks with discrete and continuously distributed delays, IEEE Trans. Neural Networks, 24 (2013), 868-877. 1

[8] X. Li, S. Song, Stabilization of Delay Systems: Delay-dependent Impulsive Control, IEEE Trans. Automat. Control, 62 (2017), 406-411. 1

[9] X. Li, J.-H. Wu, Stability of nonlinear differential systems with state-dependent delayed impulses, Automatica J. IFAC, 64 (2016), 63-69. 1

[10] X. Li, X. Zhang, S. Song, Effect of delayed impulses on input-to-state stability of nonlinear systems, Automatica J. IFAC, 76 (2017), 378-382.

[11] X. Li, Q. Zhu, D. O'Regan, pth Moment exponential stability of impulsive stochastic functional differential equations and application to control problems of NNs, J. Franklin Inst., 351 (2014), 4435-4456. 1

[12] X. Liao, Theory Methods and Application of Stability, Huazhong University of Science and Technology Publ., Wuhan, (2010). 2.1

[13] X. Liu, G. Ballinger, Uniform asymptotic stability of impulsive delay differential equations, Comput. Math. Appl., 41 (2001), 903-915. 1

[14] B. Liu, C.-X. Dou, D. J. Hill, Robust exponential input-to-state stability of impulsive systems with an application in micro-grids, Systems Control Lett., 65 (2014), 64-73. 1

[15] B. Liu, D. J. Hill, Z. J. Sun, Mixed K-Dissipativity and Stabilization to ISS for Impulsive Hybrid Systems, IEEE Transactions on Circuits and Systems II: Express Briefs, 62 (2015), 791-795. 1

[16] X. Liu, Q. Wang, Stability of nontrivial solution of delay differential equations with state-dependent impulses, Appl. Math. Comput., 174 (2006), 271-288. 1

[17] X. Liu, Q. Wang, The method of Lyapunov functionals and exponential stability of impulsive systems with time delay, Nolinear Anal., 66 (2007), 1465-1484. 1

[18] J. Lu, D. W. C. Ho, J. Cao, A unified synchronization criterion for impulsive dynamical networks, Automatica J. IFAC, 46 (2010), 1215-1221. 1

[19] I. Stamova, T. Stamov, X. Li, Global exponential stability of a class of impulsive cellular neural networks with Supremums, Internat. J. Adapt. Control Signal Process., 28 (2014), 1227-1239. 1

[20] T. Yang, Impulsive control theory, Springer, Berlin, (2001). 1, 2.2

[21] T. Yang, Impulsive Systems and Control: Theory and Application, Nova Science Publishers, New York, (2001). 1

[22] X.-S. Yang, J. Cao, J.-L. Qiu, pth moment exponential stochastic synchronization of coupled memristor-based neural networks with mixed delays via delayed impulsive control, Neural Networks, 65 (2015), 80-91. 1 